\title{
Tunnelling barriers for high temperature superconductor devices
}

\author{
G.J. Gerritsma*, M.A.J. Verhoeven, R. Moerman, D.H.A. Blank, H. Rogalla \\ University of Twente, PO Box 217, 7500 AE Enschede, Netherlands
}

\begin{abstract}
In this contribution we will discuss the requirements to be imposed on the barriers of HTS Josephson junctions as well as quasi particle injection devices. Factors that play a role are noise immunity, operating margins, injection efficiency etc., leading to the need for barriers in which the charge transport is dominated by direct tunnelling. So far, it is unclear if this has ever been observed in any practical device. We will demonstrate that localised states, that are probably ubiquitous in the materials used, are of prime importance in understanding device properties. In the model system we investigated, i.e. DyBCO/PrBCO/DyBCO, gallium-doping on the copper chain sites reduces the density of localised states appreciably without affecting the barrier height. Furthermore, it seems that due to an on-site Coulomb repulsion. pair transport proceeds via direct tunnelling, at least in the temperature region $T<T_{\text {c }} / 2$. Quasi particles, in contrast, tunnel resonantly via one or more localised states. At higher temperatures and bias voltages inelastic processes may dominate. These resonant tunnelling processes reduce the normal state resistance, and hence the $I_{c} R_{n}$-product. The consequences of these findings will be discussed and directions for future work set.
\end{abstract}

Keywords: Tunnelling barriers; High temperature superconductor devices

\section{Introduction}

Tunnelling barriers play an important role in Josephson junctions as well as in quasi=particle injection devices. Two important parameters describe the properties of these barriers. namely the critical current density and the normal state resistivity. The former results from Cooper pair tunnelling, the latter from quasisparticle tunnelling. Both depend on the properties of the barriers as well as the electrodes, but the upper limit of their product, the socalled $I_{\mathrm{v}} R_{\mathrm{n}}$-product, is eventually determined by the energy gap of the superconducting electrodes. However, in practice various factors may give rise to a considerable reduction of its ideal value. Most junctions that have been reported in literature so far have an $\boldsymbol{l}_{\mathrm{c}} \boldsymbol{R}_{\mathrm{n}}$-product of about 1 $\mathrm{mV}$ or less at $4.2 \mathrm{~K}$, a value that decreases by an order of magnitude at $77 \mathrm{~K}$. There are, however, also some exceptions even if no excess current occurs. In the latter case the value of the $I_{\mathrm{c}} \boldsymbol{R}_{\mathrm{n}}$-product is no longer directly related to intrinsic material properties. These excess currents may result from pinholes in the barrier. or other inhomogeneities, but also from flux flow. The latter can be prevented by making the size of the junctions small enough.

\footnotetext{
"Corresponding author. Tel: $(+31-53)$ 489-3122; Fax: $(+31-53) 489$. 1099: e-mail: G.J.Gerritsma(iotn.utwente.nl
}

For digital applications it can be demonstruted that critical current densities in excess of $10 \mathrm{kA} \mathrm{cm}-2$ are required to arrive at an acceptable influence of noise on the switching characteristics of circuits containing HTS Josephson junctions to be operated around about $40 \mathrm{~K}[1]$. Furthermore, it can be shown that a high $I_{\mathrm{c}} R_{\mathrm{n}}$-product is favourable for the operating margins of digital circuits and the output of analogue devices. For quasi-particle injection devices, of course, only the normal state resistance plays a role. High injection energies are obtained if its value is high, furthermore, it also determines the isolation between gate and channel of 3-terminal devices based on the injection effect and the non-equilibrium properties of the superconducting channel. However, the tunnelling process must be elastic in order to avoid energy loss in the barrier merely resulting in heating effects.

In the following discussion we will focus our attention on junctions having a high ohmic oxide barrier of approximately the same lattice parameters as the superconducting electrodes, in order to allow for fully epitaxial growth. In principle, one expects that Cooper pairs as well as quasiparticles tunnel through such a barrier and that the proximity effect is absent. This is certainly not true for all kinds of HTS Josephson junctions. This tunnelling is, however, not necessarily of a direct nature, and may very well proceed via so-called localised states in the barrier. This is well known for low- $T_{\mathrm{c}}$ cunnel junctions, and has 
been extensively investigated, especially for amorphous silicon barriers [2]. The theory of inelastic tunnelling developed by Glazman and Matveev [3] turned out to describe the observed quasi particle transport quite well in these junctions. Therefore, one might expect that this could also be the case in high- $T_{\mathrm{c}}$ junctions with an insulating barrier having a certain amount of disorder. Given the present state in the art of thin film growth, there are many defects available that could act as localised states. Their presence will open up indirect tunnelling channels via one, two, or more localised states that act in parallel with direct tunnelling and as a result the conductance of the junction will increase. This may be written as

$G=G_{0}+G_{1}+G_{2}+G_{3}+\ldots$

Here $G$ is the total conductance, $G_{0}$ the contribution from direct tunnelling, $G_{1}$ from indirect tunnelling via one localised state, $G_{2}$ from two localised states etc.. All terms are exponentially dependent on the thickness of the barrier, and we may write

$G_{n}=C_{n} \mathrm{e}^{-2 d /(n+1) a}$,

where $C_{n}$ is a prefactor that may depend on temperature and junction bias voltage, $d$ is the thickness of the barrier and $a$ is a characteristic decay length for tunnelling. In general, the first two terms are independent of temperature and bias voltage. The third and higher order terms are predominantly inelastic and hence temperature and voltage dependent. For the third and fourth term we may write

$C_{2}=C_{30} X^{4 / 3}, C_{3}=C_{30} X^{5 / 2}$.

where $X=T$ in case $e V \ll k T$, or $X=V$ if $e V>k T$, and $C_{20}, C_{30}$ are constants that depend on the details of the distribution of the locenlised states. From eq. (2) it is obvious that direct tunnelling $(n=0)$ dominates for the thinnest barriers, and that with increusing thickness indirect tunnelling processes come into play. In principle, in a given thickness range one of these processes may be the dominant charge-transport mechanism.

As we will see later, Cooper pair tunnelling via localised states is unlikely to occur in our junctions, probably due to an on-site Coulomb repulsion. If this happens, the Cooper pairs can only tunnel directly from one electrode to the other. Therefore, we will not discuss the theory for pair tunnelling via localised states. Nevertheless, the critical current may be lower than what would be expected from the energy gap, or order parameter, of the bulk electrode alone. One reason is rather fundamental and is intrinsically related to the short coherence length in a HTS superconductor. Given the fact that the order parameter in the insulating barrier should be zero, this short coherence length will result in a depression of the order parameter at the interface between superconductor and insulating barrier, as was demonstrated by Deutscher and Müller [4]. The other is also related to the short coherence length, but in a more prosaic way, i.e. if there is an interface layer that has a reduced order parameter due to defects or inter diffusion. In both cases the tunnelling Cooper pairs do not come from the bulk of the electrodes, but from a region near the interface with a reduced order parameter. As a result the critical-current density will be lowered. Therefore, in planar sandwich junctions fabricated from $c$-axis oriented electrodes, it is very difficult, although not impossible, to achieve a high $I_{\mathrm{c}} R_{\mathrm{n}}$-product [5].

So far, convincing experimental evidence is lacking for which of the mechanisms mentioned above are responsible for the relatively low $I_{\mathrm{c}} \boldsymbol{R}_{\mathrm{n}}$-products in HTS junctions. Therefore, we fabricated hundreds of junctions consisting of $\mathrm{REBa}_{2} \mathrm{Cu}_{3} \mathrm{O}_{7}$ electrodes, where $\mathrm{RE}=\mathrm{Y}$ or $\mathrm{Dy}$, and using $\mathrm{PrBa}_{2} \mathrm{Ga}_{x} \mathrm{Cu}_{3-x} \mathrm{O}_{7}$ barriers with various $\mathrm{Ga}$-doping levels $x$ and thickness $d$. In the analysis of the charge transport only those junctions were used having a negligible amount of excess current. The technology we used is that of the ramp-type junction that, since its inception in the spring of 1990 [6], has been considerably improved in recent years.

In Section 2 of this article we will briefly analyse the growth of thin barriers on ramps and discuss its consequences. More details can be found in an article by Verhoeven et al. [7]. This will be followed by a section devoted to the analysis of the measured $I$-characteristics of these junctions. Finally we will draw some conclusions from the results obtained thus far.

\section{Peculiarities of ramp structures}

Epituxial films with the c-axis perpendicular to the substrate surface of $(001) \mathrm{SrTiO}_{3}$ have been deposited using off-axis r.f.amagnetron sputtering in a fairly conven= tional way. Substrates were glued with silver paint to a stainless heater block and out gassed in ambient at $150^{\circ} \mathrm{C}$. Next this block was brought into the sputter chamber via a load-lock system, and out gassing was continued at the deposition temperature, about $770^{\circ} \mathrm{C}$. until the background pressure of $10^{-6}$ mbar was achieved. As sputtering gas we used a 1:1 mixture of $\mathrm{Ar:O} \mathrm{O}_{2}$ at a pressure in the range of 10-20 Pa. Deposition conditions were changed to optimise the growth of each layer separately. In order to increase the reproducibility of the layer growth, we added about $1 \%$ of water vapour to the sputter gas. Due to the increased reactivity of the sputter gas this probably prevents the target from deoxygenating in the course of time [8]. The junction fabrication process is started with the deposition of the base electrode material followed by an insulating layer, usually from the same material as the barrier. Ramps are being etched by a $500 \mathrm{eV}$ argon-ion beam from a Kaufmann source, using a resist stencil to define its position. The beam hits the sample under an angle of $45^{\circ}$, whereas the actual ramp angle is about $20^{\circ}$ with the substrate surface. After removal of the resist the ramps are 
cleaned with the ion beam, followed in situ by the deposition of barrier layer and counter electrode. As was already pointed out by Hunt et al. [9] ion-beam milling at energies $\gg 50 \mathrm{eV}$ probably results in the formation of the non-metallic cubic YBCO phase, and recently Jia et al. [10] demonstrated with HREM analysis, that this layer could be $4 \mathrm{~nm}$ thick. In order to assess the influence of etching damage on the electrical transport properties we fabricated several junctions without barrier. The critical current density of these ramp contacts at various cleaning voltages is depicted in Fig. I. A considerable reduction in critical current density may result from etching, but for an ion-beam voltage of $50 \mathrm{eV}$ ion the effect is only marginal and unobservable with HREM.

Using this impreved cleaning procedure, we investigated the growth of thin PBCO barrier layers on these ramps by atomic force microsc, (AFM). The micrographs were taken in open air with a anoscope III. Commercial $\mathrm{Si}_{3} \mathbf{N}_{4}$ tips were used. All micrographs were recorded with at least two different tips, and checked at different scan angles to rule out artifacts caused by tip profile. In order to enhance the details of the surface structure, we subtracted the smoothed ramps from the measurements. From these measurements it became evident that the bare ramp has a more or less faceted, terrace-like structure. The steps are of unit-cell height. i.e. about $1 \mathrm{~nm}$. This does not change when a ramp is annealed at the deposition temperature for approximately one hour. In this example the ramp lies parallel to a crystallographic axis of the substrate. If this is not the case terraces are broke; up and more facets appear. For detailed analyses of the growth on ramps we refer to Verhoeven et al. 17]. In Fig. 2 and Fig. 3 we compare superimposed linescans of bare ramps. with a nominally o $\mathrm{nm}$ barrier on top. with those having a $10 \mathrm{~nm}$ batrier on top. As is evident from these ligures, junctions with a bafrier of $6 \mathrm{~nm}$ and below are likely to have pinholes. Barriers of thickness $10 \mathrm{~nm}$ and above are free of pinholes and reasonably smooth. The latter results in negligible excess current and well defined Fraunhofer pattern. During

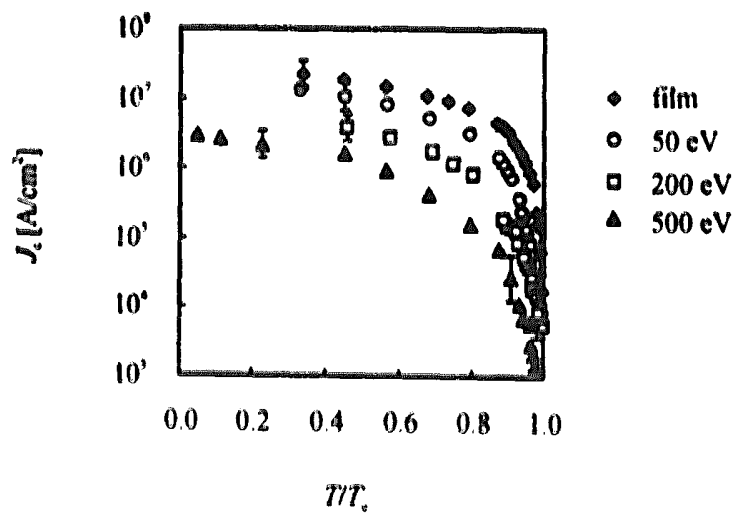

Fig. I. Critical current density as a function of reduced temperature for a thin film and ramp contacts fabricated with various ion-beam cleaning
voltages.

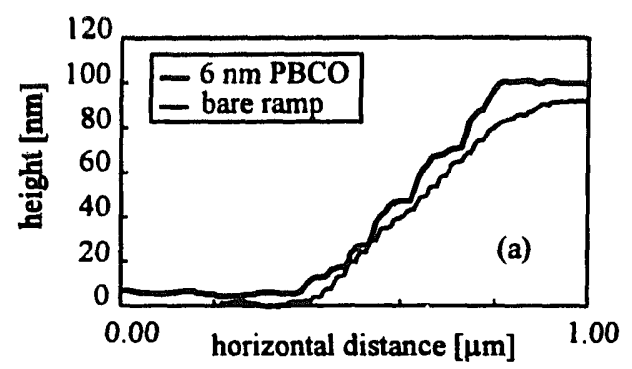

Fig. 2. AFM line scans of bare ramp and ramp covered with a nominally $6 \mathrm{~nm}$ PBCO barrier. Barriers are inhomogeneous and may contain pinholes.

the first $6 \mathrm{~nm}$ of growth the surface roughness increases, which is probably due to the anisotropy in the growth rate along the $c$-axis. respectively the $a b$-plane, direction. Thereafter, the ramps become smoother with increasing barrier thickness. This is probably because now the newly formed deep trenches act as secondary nucleation sites.

As a result it will be impossible to increase the $I_{\mathrm{c}} R_{\mathrm{n}}$ product of these junctions merely by reducing the barrier thickness. Either a barrier material should be used that is more isotropic or the deposition conditions should be modified in such a way that initial growth starts from many nuclei and that the islands quickly coalesce before they significantly roughen the surface due the anisotropic growth rates. If a different material is required in order to decrease the barrier thickness. its characteristic tunnelling distance should not be shorter than that of PBCO. Otherwise. an increase in barrier layer homogeneity may be offset by the fact that an even thinner layer would be necessary.

\section{Burrier doping}

When we started studying the influence of doping the barrier material on the $I V$-characteristics of Josephson junctions, it was quite well known that doping PBCO with gallium leads to an increased resistivity of the bulk material. Up to doping levels of $x=0.1$ or more, the gallium atoms preferentially substitute for the copper atoms in the CuO-chains [11]. Furthermore, it is generally assumed that the cherge transport in PBCO takes place in

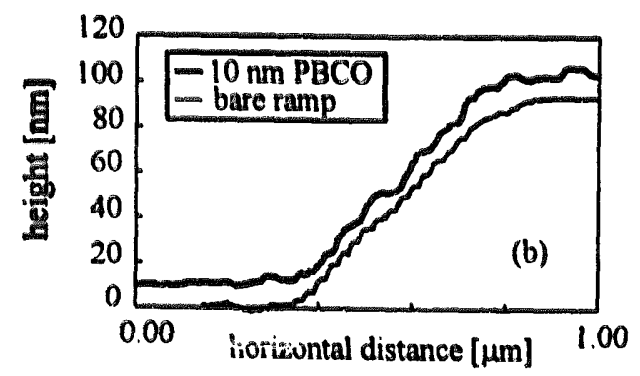

Fig. 3. AFM line scans of hare ramp and ramp covered with a nominally $10 \mathrm{~nm}$ PBCO barrier. Barriers are homogeneous and free of pinholes. 
the form of VRH [12]. What we expected, of course, was an increase in junction resistance, but we did not know what would happen to the critical current.

In the sequel we will present the data on the normal state conductance and critical-current density of the junctions in more detail. In Fig. 4 the criticai current density of the junctions at two temperatures and two Ga-doping levels are presented as a function of barrier thickness. The value for the barrier thickness that we use is the nominal thickness in the direction perpendicular to the substrate surface as inferred from the calibrated growth rate. This is not the actual distance travelled by the tunnelling particles, but we simply do not know in which direction tunnelling takes place. In any case, all decay lengths that we obtain from the measurements are mutually comparable. What is immediately evident from the measurements is the fact that, much to our surprise, the critical current density does not depend on Ga-doping. This also holds for the decay length. Furthermore, the decay length is independent of temperature, at least for temperatures below about $T_{\mathrm{c}} / 2$. Zero doping is not shown in this figure, but these data do not change our findings. In Fig. 5 the conductance $G$ is depicted as a function of the barrier thickness for 3 Ga-doping levels at $4.2 \mathrm{~K}$. From these data it is evident that $G$ decreases with increasing doping, as expected, but the slope, i.e. the decay length, remains constant. Also, a striking feature is the fact that the Cooper pair decay, or coherence, length $\boldsymbol{\xi}$ and the quasi particle decay length differ by a factor of two. This is exactly what one would expect if the former particles are tunnelling directly. whereas the latter are tunnelling indirectly via one localised state at $4.2 \mathrm{~K}$. The fact that the Cooper pairs tunnel directly is also supported by the fact thit $\xi$ is independent of temperature. In the case of a proximity effect this would not be true. The effect of Ga-doping, assuming the theory of Glazman and Matveev to be applicable, leads to a

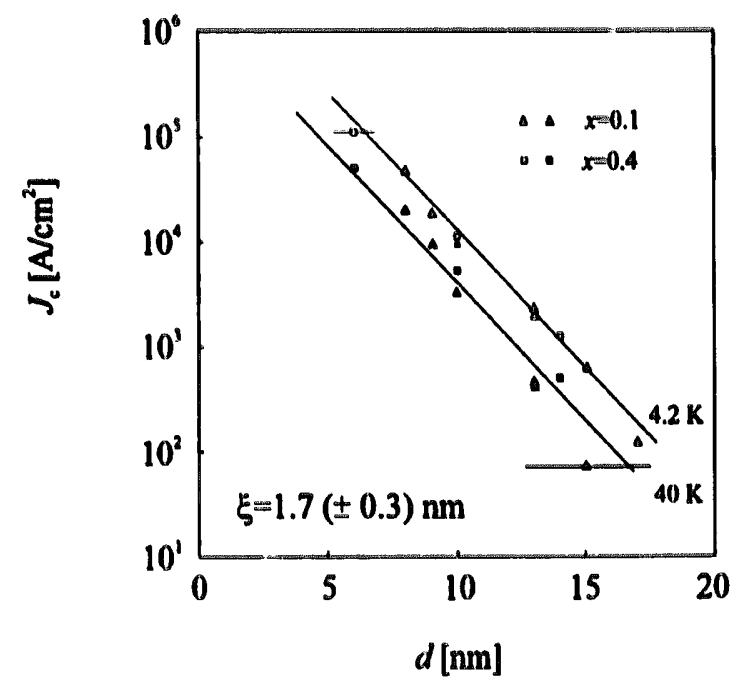

Fig. 4. Critical current density as a function of nominal barrier thickness at two temperatures and two Ga-doping levels.

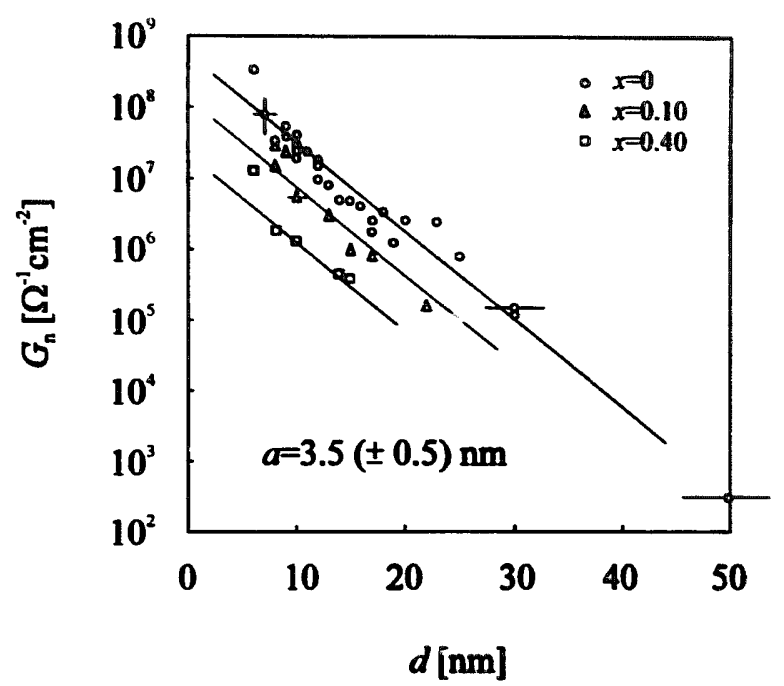

Fig. 5. Conductance as a function cf nominal barrier thickness at $4.2 \mathrm{~K}$ and 3 Ga-doping levels.

reduction in the density of localised states near the Fermi energy surface. Further evidence that this theory may hold comes from the fact that for PBCO we also observed that $G-G_{\text {lin. }}$, i.e. the difference between the total conductance and the linear part, is proportional to $T^{4 / 3}$ at low bias voltages and to $V^{4 / 3}$ at high oias voltages. This is just what is predicted by Glazman and Matveev for $G_{2}$ and results from inelastic tunnelling via two localised states. From this evidence we conclude that localised states in the barrier play an important role in the observed charge-transport processes in our junctions.

The microscopic origin of these states is presently unknown. but their density is reduced by Ga-doping in the CuO-chains. The answer to this question is probably intimately connected with the absence of superconductivi ty, or even metallic conductivity, in PBCO. In an article by Takenaka et al. $|13|$ in which polarised optical reflectivity spectra of untwinned $\mathrm{PBCO}$ in the range $0.03=40 \mathrm{eV}$ are discussed, it was demonstrated that holes reside mainly in the CuO-chains (50\%) and at the $\mathrm{Pr}$-atom sites $(50 \%)$. The latter is not a direct consequence of the measurements. but results from a charge-neutrality argument. The tendency for hole doping in the chains, instead of the planes, also finds support from theory [14.15]. In the last reference it is demonstrated that holes from the planes may be grabbed by the so-called FR-band. The latter is assumed to be very sensitive to $\mathrm{Pr}$ disorder and as a result will be nonconducting. Furthermore. Takenaka et al. observed a decrease in the conductivity along the $\mathrm{CuO}$-chain direction below $0.2 \mathrm{eV}$, an indication of localisation of chain holes caused by disorder. The latter probably results from chainoxygen defects. These observations are consistent with the observed semiconductive resistivity of this compound. Hence, we think it to be quite likely that if Cu-atoms with formal valence state $2.5+$ are replaced by Ga-atoms with valence state $3+$, the number of holes will be reduced. 
This in turn leads to a shift of the Fermi level away from the mobility edge and hence a reduction of the density of localised states near the Fermi level. From the decay length we infer a tunnelling barrier height of $3 \mathrm{meV}$, a rather low value. Here we assumed that the particles have an effective mass equal to that of the free electron. There is good reason to assume that the effective mass should be much lower and hence the barrier higher. This is further supported by the observation that tunnelling occurs up to 10 $\mathrm{mV}$ or higher bias voltage. Given the properties of the Cooper pairs and the localised states, an on-site Coulomb repulsion may prevent the super current tunneling via the localised states.

\section{Conclusions}

As has been demonstrated, charge transport in ti.'S ramp-type Josephson junctions with a Ga-doped PBCU barrier is dominated by tunnelling processes. The Cooper pairs tunnel directly, at least for temperatures below about $T_{1} / 2$, whereas the quasiparticles tunnel indirectly via localised states. The density of these states may be reduced by Ga-doping, which we tested so far up to $x=0.4$. As this does not affect the Cooper-pair tunnelling. doping results in an increase of the $l_{\mathrm{c}} R_{\mathrm{n}}$-product of these junctions. Presently we are able to fabricate junctions with an $I_{\mathrm{c}} R_{\mathrm{n}}$ " product up to about $10 \mathrm{mV}$. Furthermore, critical-current densities of $10 \mathrm{kA} \mathrm{cm}^{-3}$ are easily achieved. Hence, these junctions may be operated at $42 \mathrm{~K}$ with the same noiseimmunity level as ordinary $\mathrm{Nb} / \mathrm{Alox}$ tunnel junctions at $4.2 \mathrm{~K}$.

The question now rises if further improvement is possible. i.e. if all particles could be forced to tunnel directly. This requires either a reduction in the barrier thickness or in their density of localised states. Therefore, growth studies of barriers on ramps are of prime importance in order to find conditions that allow for a smooth deposition of the thinnest barriers. This is not necessarily restricted to PBCO. There are four 1:2:3-compounds which allow for a $100 \%$ substitution of their Cu-chain atoms by gallium, and yet reveal single-phase formation. If Codoping is used there are even 10 single-phase compounds available [16].

\section{Acknowledgments}

This research is in part supported by the EC, contract number ESPRIT 6677.

\section{References}

(1) R.J. Wiegerink, G.J. Gerritsma, E.M.C.M. Reuvekamp, M.A.J. Verhoeven and H. Rogalla, IEEE Trans. . Ifpl. Supercond., 5 (1995) 3452.

[2] Y. Xu, D. Ephron and M.R. Beasly, Phys. Rev. B. 52 (1995) 2843.

[3] L.I. Glazman and K.A. Matveev, Sov. Phys. JETP. 67 (1988) 1276; Zh. Eksp. Teor. Fiz.. 94 (1988) 332.

[4] G. Deutscher and K.A. Müller, Phys. Rev. Lett., 59 (1987) 1745.

[5] I. Bozovic and J.N. Eckstein, J. Supercond., 8 (1995) 537.

[6] J. Gao, W.A.M. Aurnink, G.J. Gerritsma and H. Rogalla, Physica $C$. $I 7 I(1990) 126$

17] M.A.J. Verhoeven, R. Moerman, M.E. Bijlsma, A.J.H.M. Rijnders, D.H.A. Blank. G.J. Gerritsma and H. Rogalla, Appl. Phys. Lett., 68 (1996) 1276.

|8| J.R. Gavaler, J. Talvacchio, T.T. Braggings, M.G. Forrester and J. Greggi, J. Appl. Phys.. 70 (1991) 4383.

$|9|$ B.D. Hunt, J.B. Barner, M.C. Foote and R.P. Vasquez. Proc. ISEC 93 Comf.. 199.3, p. 221.

|I(0) C.L. Jia. M.I. Faley, U. Poppe and K. Urban, Appl. Phys. Letr. 67 (1995) 3635.

IIII E. Sodtke. C. Andrzejak, D. Guggi and Y Xu, Physica C. 180 (1991) 50 .

112| B. Fisher, G. Koren. J. Genossar, L. Patlagan und E.L. Gartstein. Mllewica C. 176 (1991) 75 .

$113 \mid$ K. Tukenaka. Y. Imanuka, K. Tumasaku, T. Itoh and S. Uehidu, Phys. Rev. B. $\$ 6$ (1902) 5833 .

|14| R. Pehrenbacher and T.M. Rice. Phys. Kev. Lett. 70 (1991) 347.

|15| A.I Lichtenstein and I.I. Mazin, Physs. Rev. Lett., 7 (1995) 1000.

|l(6) T.A. Mary, N.R.S. Kumar and U.V. Varadaraju, J. Solid State Chem., 107 (1993) 524 